\title{
Advanced and Innovative Optimization Techniques in Controllers: A Comprehensive Review
}

\author{
Aditya Tiwary \\ Department of Fire Technology \& Safety EngineeringInstitute of Engineering \& Science, IPS Academy Indore (M.P.), India \\ adityatiwary0217@gmail.com
}

Abstract - New commercial power electronic controllers come to the market almost every day to help improve electronic circuit and system performance and efficiency. In DC-DC switching-mode converters, a simple and elegant hysteretic controller is used to regulate the basic buck, boost and buck-boost converters under slightly different configurations. In AC-DC converters, the input current shaping for power factor correction posts a constraint. But, several brilliant commercial controllers are demonstrated for boost and fly back converters to achieve almost perfect power factor correction. In this paper a comprehensive review of the various advanced optimization techniques used in power electronic controllers is presented.

Keywords- power electronic controllers; optimization techniques; AC-DC converters; Ant Colony Optimization

\section{INTRODUCTION}

Power electronics converters are generally consist of only semiconductor switches and energy storage elements. Nonisolated converters are often preferred in applications thatelectrical isolation is not a necessity, because they are less bulky and costly, and more efficient and reliable. On the otherhand, isolated power converters often use either transformer or coupled inductor for multiple purposes such as voltage level shifting, obtaining multiple outputs, providing galvanic isolation and ground loop avoidance. The AC transformer in converters needs an AC voltage or a square/quasi square wave voltage at the primary side for proper operation and saturation avoidance. A transformer can be used in buck type converters to allow voltage step-up feature. In addition, a simple way to achieve multiple outputs in a single converter is to use multiple windings transformers or coupled inductors. Moreover, employing transformers in the circuit allows electrical isolation that provide galvanic isolation between the input and output modules of a converter. When two or more electrical devices share a common ground in a power system, the current from the return path of one device can disrupt the operation of otherdevices, which can be avoided by utilizing transformers.

\section{LITERATURE REVIEW}

In this paper, the simulation results of static var compensator (SVC) for improvement of transient stability in a photo voltaic (PV) system integrated power system are presented [1]. Blondin et al. [2] proposed an optimal gain tuning method for PID controllers is proposed using a novel combination of a simplified Ant Colony Optimization algorithm and Nelder-Mead method (ACO-NM) including a new procedure to constrain NM. Moghadam et al. [3] proposes an optimization-based tuning methodology for real and complex Fractional-Order Proportional-Integral (FOPI) controllers. An optimized Mamdani-type hybrid Fuzzy P+D controller was proposed by Soylu et al. [4]. In this work a Generalized type-2 Fuzzy Logic System (GT2FLS) approach for dynamic parameter adaptation in metaheuristics and for optimal fuzzy controller design is presented by Castillo and Angulo [5]. The optimal tuning of adaptive flap controller canimprove adaptive flap control performance on uncertain operating environments, but the optimization process is usually time-consuming and it is difficult to design proper optimal tuning strategy for the flap control system (FCS). To solve this problem, a novel adaptive flap controller is designed based on a high-efficient differential evolution (DE) identification technique and composite adaptive internal model control (CAIMC) strategy by $\mathrm{Li}$ et al. [6]. This paper deals with Automatic Generation Control (AGC) of three area multi source interconnected power system assimilating Battery Energy Storage System (BESS). The plant is designed with Particle Swarm Optimization (PSO)tuned Integral controller (IC) which is carried out on three different sources, thermal, gas and hydro interconnected power system with and without BESS [7].

Guo and Scherer [8] proposed an exact state-space solution for the problem of designing a robust gain-scheduled controllerwith a hierarchical structure and based on semi-definite programming. Kumar et al. [9] proposed optimized controller applied to a three storey prototype structure fixed with an MR damper. This structure has been subjected to various seismic events for performance evaluation. This paper proposes a novel technique to design a PID control using virtual sensing system, consisting of Diagonal Recurrent Neural Network (DRNN) and Extended Kalman Filter (EKF), which predicts the immeasurable states of the quadrator system based on the current states and control inputs [10]. This article presents a novel tuning design of Proportional-IntegralDerivative (PID) controller in the Automatic Voltage Regulator (AVR) system by using Cuckoo Search (CS) algorithm with a new time domain performance criterion. This performance criterion waschosen to minimize the maximum overshoot, rise time, settling time and steady state error of the terminal voltage. In order to 
compare CS with other evolutionary algorithms, the proposed objective function was used in Particle Swarm Optimization (PSO) and Artificial Bee Colony (ABC) algorithms for PID design of the AVR system [11]. Laware et al. [12] accords the level control of single-input-single-output (SISO) level control system based on the fusion of sliding mode control (SMC) and evolutionary techniques or bio-inspired techniques. The nondominated sorting genetic algorithm II (NSGA-II) and multiobjective particle swarm optimization (MOPSO) are considered as two evolutionary techniques. Here, a comparative analysis of performances of an optimal proportional-integral (PI) controller, proportional-integral-derivative (PID) controller, conventional SMC, NSGA-II based tuned SMC and SMC parameter tuning using MOPSO algorithm has been carried out through MATLAB/SIMULINK. In this paper, the authors analyzed and compare three different MDO approaches considering the tuning of a Proportional-Integral (PI) controller and plant design simultaneously. As conflicting objectives might appear, we compare such approaches using multi- objective optimization [13].

Kuti and Galambos [14] investigate the computer-regulated propofol administration in anesthesia during medical interventions considering output feedback and robust PID control. The paper applies the Affine Tensor Product Model Transformation to derive the appropriate polytopic quasi-LPV representation of the closedloop dynamics. Khan et al. [15] work is concerned with controlling the dissolved oxygen in a biological fermenter using a PID (Proportional-Integral- Derivative) Controller. The product from this fermenter is used to create the immunization for a medical illness. Enrique et al.

[16] proposes an scheduled-gain PID Controller and implemented in a micro-controller to accurately adjust the amount of weight on the bit (WOB) and avoid disturbances. High-frequency data was acquired using LabVIEW and analyzed in real time through the MATLAB programming environment. The parameter optimization method for multivariable systems is extended to the controller design problems for multiple input multiple output (MIMO) square fractional-order plants. The algorithm can be applied to search for the optimal parameters of integer-order controllers for fractionalorder plants with or without time delays Xue et al. [17]. Kang and Yan [18] proposed design of the controller transformed into a convex optimization problem, which is tackled by means of the appropriate optimization method. In this paper, authors have designed and test a primary-secondary user resource-management controller in cognitive radio vehicular networks, under hard and soft collision constraints. The resource-management problem into a stochastic network utility maximization problem and derive the optimal steady- state controllers, which adaptively allocate the access time- windows to the secondary-users [19]. In this work, an optimal control for a full-car electromechanical active suspension is presented by Haemers et al. [20]. In this paper, a novel evolution (MDE) algorithm optimized fuzzy PID controller is proposed for load frequency control (LFC) of interconnected power system with the consideration of nonlinearity. The gains of the fuzzy PID controller are optimized using different strategies of DE algorithm. Then, modification in DE algorithmis proposed for the best strategy by a simple but effective scheme of changing two of its most important control parameters (step size and crossover probability) with an objective of achieving improved performance [21]. Dynamic modeling and control system design for the hose-drogue system (HDS) in the docking stage of autonomous aerial refueling (AAR) are investigated in this paper by Sun et al. [22]. The authors parameterize all the controllers leading to the given performance specifications (cross-over freq, phase margin, iso-damping) and then do the optimization of the cross- over freq and phase margin to achieve the best step-response performance [23].

Energy storage systems have great potential in maintaining the power balance and sustaining the grid frequency during sudden disturbances to support the automatic generation control in a power system. Hence, the role of a capacitive energy storage unit as an energy storage device and a thyristor- controlled phase shifter in the automatic generation control of a two-area interconnected power system having multiple gas- hydro-thermal mixed generating units in a deregulated power environment has been investigated in this paper by Dhundhara et al. [24]. Frequency regulation of power systems by fuzzy aided PID controller is proposed in this study. The controller gains are optimized by an Improved Grey Wolf Optimization (IGWO) method. The improvement in GWO method is done by an approach which does not consider less important delta wolves for updating the position vector in the hunting stages of the algorithm thus making the algorithm simpler with less implementation time. Proposed IGWO is initially evaluated using some standard test functions. The outcomes are equated with GWO, modified GWO (MGWO), Differential Evolution (DE), Gravitational Search Algorithm (GSA) and Particle Swarm Optimization (PSO) techniques to illustrate its supremacy [25]. This paper provides brief design procedure of fractional order proportional-integral-derivative (FO-PID) controller through the indirect approach of approximation using constant phase technique. The new modified dynamic particle swarm optimization (IdPSO) technique is proposed to find controller parameters [26]. Sahu et al. [27] deals with load frequency control (LFC) in an islanded two area AC micro grid (MC) system. In this study the proposed two area MG system comprises different micro sources including Microturbine (MT), Diesel engine generator (DEG) and Fuel Cells (FC) which are primarily responsible for balancing load and power generation in an interconnected system. MG in grid-connectedmode has lower possibility of frequency control problem due to active presence of utility grid. Tashin et al. [28] highlights the attempt of incorporating geothermal power plant (GTPP), dish-Stirling solar thermal system (DSTS) and high voltage direct current transmission (HVDC) link, with the conventional thermal system, in automatic generation control of an interconnected power system under deregulated environment. Appropriate generation rate constraints are provided in thermal systems. This paper proposes the development of constrained next generation RTDA controller to handle various linear inequality constraints in a multi-variable control framework [29]. This work proposes a tuning parameters optimization approach for Dynamical Sliding Mode Controllers developed from a reduced model using optimization criteria and restrictions which are given by performance factors that the process must reach by Baez et al. [30].

In this paper, the MFO-based design of blade pitch controllers (BPCs) is proposed for wind energy conversion system (WECS) to enhance the damping of oscillations in the output power and voltage. The simple Proportional-Integral- Differential (PID) is used to realize the advantage of the proposed hybrid referential integrity MFO technique by Ebrahim et al. [31]. In this article, Integral - Proportional Derivative (I-PD) controller has been 
designed and implemented in simulation and real time for the Magnetic levitation (Maglev) system which is both nonlinear and unstable in nature. The nonlinear Maglev plant has been linearized around the equilibrium point to obtain the linearized model transfer function. The objective function, which has been formulated by taking the modulus of the characteristic polynomial of the plant along with the controller at dominant pole location, has been minimized using the recently evolved Jaya algorithm [32]. Lu et al. [33] proposes a self-adaptive state-space predictive functional control (APFC) based on extremal optimization method to design PID controller called EO-APFC-PID, wherein, the self-adaptive means, i.e., a forgetting factor recursive least squares (FFRLS) mechanism is embedded into state-space predictive functional control (PFC), and the proposed EO is exploited to alleviate the challenging problem that the elements in weighting factors of APFC technique are lacking analytical knowledge. Nayak et al. [34] proposes a maiden comparative performance analysis of interval type-2 fuzzy-PID controller with \& without derivative filter (T2FPIDF \& T2FPID), type-1 fuzzyPID controller (T1FPID) and conventional PID controller for Automatic Generation Control (AGC) in a two area interconnected thermal power system is presented. The proposed controllers are optimally designed using a novel adaptive symbiotic organism search (ASOS) \& symbiotic organism search (SOS) optimization techniques. In this paper, authors have presented method for designing Fuzzy controller rule base using a new swarm intelligence algorithm, which is based on the Bat algorithm. The Bat algorithm is one of the most recent swarm intelligence based algorithms that simulates the intelligent hunting behavior of the bats found in nature. The main objective is to design the fuzzy rule base of fuzzy controller respecting the desired performance [35].

Farajdadian et al. [36] proposed a fuzzy logic controller (FLC) for MPPT in photovoltaic systems. Four optimization algorithms are presented in this paper for optimizing fuzzy membership functions (MFs) and generating proper duty cycle for MPPT. This paper presents a novel contribution of a low complexity control scheme for voltage control of a dynamic voltage restorer (DVR). The scheme proposed utilizes an error- driven proportionalintegral-derivative (PID) controller to guarantee better power quality performance in terms of voltage enhancement and stabilization of the buses, energy efficient utilization, and harmonic distortion reduction in a distribution network [37]. Lee et al. [38] presents the development of a self-tuned HVAC controller that provides customized thermal conditions to satisfy occupant preferences (i.e., online learning) while minimizing energy consumption, and the implementation of this controller in a real occupied office space. The evolution of personalized thermal preference models and the delivery of thermal conditions with model predictive control (MPC) form a closed-loop. Ahmed et al. [39] presents an adaptive controller for grid-tie DC-AC inverter in grid-connected Photovoltaic (PV) power system supplying a pulse AC load. The proposed controller oversees regulating the dc-bus voltage, managing the injected power to the grid, and minimizing the injected harmonics. Blondin et al. [40] proposes a new optimization framework using a simple performance criterion to achieve rapid time response characteristics as well as to meet robustness and stability requirements. The performance of the approach is confirmed by comparing its results to those obtained with two previously published performance criteria and the results obtained with the systune command in Matlab and the MathWorks PID tuning algorithm. This paper presents an $\mathrm{N}-1$ multicontingency AC optimal power flow (OPF) thatembeds, in the same mathematical optimization model, a set of transient stability constraints (TSC) that guarantee rotor angle and angular velocity variation within technical limits, at given $\mathrm{N}-1$ contingencies. Furthermore, the proposed model considers the operation of volt/var controllers, such as shunt elements and OLTC transformers, to further improve the operation of the EPS, under given levels of demand and generation [41]. Zamzoum et al. [42] proposed an adaptive controller based onfuzzy logic concept is proposed to limit the extracted power atrated value with improving its quality and to mitigate the loads applied on the turbine and the drive train in full loads conditions by adjusting the pitch angle. This paper aims to provide a review of different controllers utilized in traditional as well as renewable energy-based power system for LFM such as; classical controllers, fractional order controllers, cascaded controllers, sliding mode controller (SMC), tilt-integral- derivative controllers, H-infinity controller and other recently developed controllers [43]. A novel Grasshopper optimized fuzzy logic control (FLC) approach based MPPT technique is proposed in this paper. In this proposed MPPT, grasshopper optimization is used to tune the membership functions (MFs) of FLC to handle all uncertainties caused by variable irradiances and temperatures. The performance of the proposed grasshopper optimized FLC based MPPT is studied under rapidly changing irradiance and temperature [44].

Jagatheesan et al. [45] proposed multi-area (Five areas: area 1, area 2 , area 3 , area 4 and area 5) reheat thermal power systems are considered with proportional-integral-derivative (PID) controller as a supplementary controller. This paper presents a quantum lightning search algorithm (QLSA) -based optimization technique for controlling speed of the induction motor (IM) drive. The developed QLSA is implemented in fuzzy logic controller to generate suitable input and output fuzzy membership function for IM drive speed controller [46]. Pajares et al. [47] present the adjustment of controller parameters using multi objective optimization techniques. This study performs a comparison study on PI cruise controller tuning for an off-road electric vehicle. A cost function is designed to reach an accurate EV speed tracking while considering safety aspects, such as no reverse speed. The ACO- NM algorithm has been demonstrated to be the most efficient compared to GA, ALO, DE, and PSO. Indeed, ACO-NM reached high-quality solutions for lower computational cost for three driving cycles by Blondin et al. [48]. A voltage-frequency management technique is proposed in this paper to retain these quantities within acceptable limits in remote islanded microgrids (MGs). The proposed technique gets activated when existing techniques that control the energy storages or adjust the set-points of generators are not successful, Arefi et al. [49]. In this study, a high order differential feedback controller (HODFC) and a developed fractional high order differential feedback controller (FHODFC) are proposed for LFC problem in multi-area power systems for the first time. The gains of the HODFC and FHODFC are optimally tuned by particle swarm optimization (PSO) algorithm aiming to minimize integral of time weighted absolute error (ITAE) performance index by Sahin et al. [50]. Ramya et al. [51] focuses on adaptive controller design using fuzzy Logic (FL) controller to get betterdynamic performance under non-linearities and parameter uncertainties. In the proposed controller, the membership function (MF) parameters are tuned in line with the 
parameter variations of the motor. In this study, a maiden application of cascade proportional-integral-derivative with filter (PIDF) and one plus fractional-order derivative (1+FOD), i.e. PIDF(1+FOD) controller is proposed for the load frequency control mechanism. The main purpose of the cascade and fractional order controller is to increase the degree of freedomand reject the disturbances faster. A novel attempt has also been made to model a static synchronous series compensator (SSSC) with AC tie-line and HVDC tie-line, Prakash et al.[52]. Mishra et al. [53] presents a Hybrid Shunt Active Power Filter (HSAPF) optimized by hybrid Particle Swarm Optimization-Grey Wolf Optimization (PSO-GWO) and Fractional Order ProportionalIntegral-Derivative Controller (FOPIDC) for reactive power and harmonic compensation under balance and unbalance loading conditions.

Soares et al. [54] presents an optimization strategy for designing an offline light-emitting diode (LED) driver with a wide-bandwidth controller aimed at reducing the converter storage capacitance. The approach is based on the modeling of all the designing process as a nonlinear constrained optimization problem so that the power circuit elements and also the controller are designed, simultaneously in order to optimize a given parameter, such as the filtering capacitance or the total harmonic distortion. This work proposes a computational methodology for coordinated tuning of the power system controllers based on Genetic Algorithm which acts maximizing simultaneously two objective functions, representing each of them the damping of electromechanical oscillations and the improvement of the automatic voltage regulator responses, considering at the same time several critical operating conditions [55]. Holt et al. [56] deals with a novel line-voltage-regulator and its power quality optimized control. Taha et al. [57] contributes to the tuning issue associated with VOC in three key points. Firstly, an algebraic solution (i.e. PIparameters) based on the first-order-plus-time- delay (FOPTD) approximations of the plant dynamics is developed, along with approximating relationships and methodology. Movahedi et al. [58] presents the effects of three FACTS controllers, static synchronous series compensator (SSSC), thyristor-controlled series compensator (TCSC), and static synchronous compensator (STATCOM), on the transient stability of a multi-machine power system in the presence of two $200 \mathrm{MW}$ wind farms based on a doubly fed induction generator (DFIG) and a $120 \mathrm{MW}$ photovoltaic (PV) solar plant are studied.

\section{CONCLUSION}

Power electronic converters allow efficient conversion and control of electrical power. Pulse width modulated converters offer better operating characteristics than the phase-controlled ones, but the very process of high-frequency switching creates undesirable side effects of its own. It seems that phase- controlled rectifiers and ac voltage controllers will maintain their presence in power electronics for years to come. This paper has described a comprehensive review of the various advanced optimization techniques used in power electronic controllers for obtaining maximum control and better efficiencyas well as reliability.

\section{REFERENCES}

[1] Shiba R. Paital, Prakash K. Ray, Asit Mohanty, Sambit Dash, "Stability improvement in solar PV integrated power system using quasi-differential search optimized SVC controller", Optik, vol. 170, pp. 420-430, 2018.

[2] M. J. Blondin, J. Sanchis, P. Sicard, J. M. Herrero, "New optimal controller tuning method for an AVR system using a simplified Ant Colony Optimization with a new constrained Nelder-Mead algorithm", Applied Soft Computing, vol. 62, pp. 216-229, 2018.

[3] M. G. Moghadam, F. Padula, L. Ntogramatzidis, "Tuning and performance assessment of complex fractional-order PI controllers", IFACPapersOnLine, vol. 51, Issue 4, pp. 757-762, 2018.

[4] S. Soylu, K. Danisman, "In silico testing of optimized Fuzzy P+D controller for artificial pancreas", Biocybernetics and Biomedical Engineering, vol. 38, Issue 2, pp. 399-408, 2018.

[5] O. Castillo, L. Amador-Angulo, "A generalized type-2 fuzzy logic approach for dynamic parameter adaptation in bee colony optimization applied to fuzzy controller design", Information Sciences, vol. 460-461, pp. 476-496, 2018

[6] N. Li, A. Mu, X. Yang, K. T. Magar, C. Liu, "A novel composite adaptive flap controller design by a high-efficient modified differential evolution identification approach", ISA Transactions, vol. 76, pp. 197-215, 2018.

[7] N. Gupta, N. Kumar, "Particle Swarm Optimization based Automatic Generation Control of Interconnected Power System incorporating Battery Energy Storage System", Procedia Computer Science, vol. 132, pp. 1562$1569,2018$.

[8] Y. Guo, C. W. Scherer, "Robust Gain-scheduled Controller Design with a Hierarchical Structure", IFAC-PapersOnLine, vol. 51, Issue 25, pp. 228233, 2018.

[9] Gaurav Kumar, Ashok Kumar, R.S. Jakka, "The particle swarm modified quasi bang-bang controller for seismic vibration control", Ocean Engineering, vol. 166, pp. 105-116, 2018.

[10] Yul Y. Nazaruddin, Angela Dian Andrini, Boby Anditio, "PSO Based PID Controller for Quadrotor with Virtual Sensor", IFAC- PapersOnLine, vol. 51, Issue 4, pp. 358-363, 2018.

[11] Zafer Bingul, Oguzhan Karahan, "A novel performance criterion approach to optimum design of PID controller using cuckoo search algorithm for AVR system", Journal of the Franklin Institute, vol. 355, Issue 13, pp. 5534-5559, 2018.

[12] A.R. Laware, D.B. Talange, V.S. Bandal, "Evolutionary optimization of sliding mode controller for level control system", ISA Transactions, vol. 83, pp. 199-213, 2018.

[13] Gilberto Reynoso-Meza, Helem Sabina Sánchez, "Multidisciplinary optimisation and controller tuning: an analysis with multi-objective techniques", IFAC-PapersOnLine, vol. 51, Issue 4, pp. 280-285, 2018.

[14] József Kuti, Péter Galambos, "Tensor Product model based PID controller optimisation for propofol administration", IFAC- PapersOnLine, vol. 51, Issue 4, pp. 645-650, 2018.

[15] Omar Khan, Chandra Mouli R. Madhuranthakam, Peter Douglas, Heron Lau, Jacob Sun, Patrick Farrell, "Optimized PID controller for an industrial biological fermentation process", Journal of Process Control, vol. 71, pp. 75-89, 2018.

[16] Enrique Z. Losoya, Eduardo Gildin, Samuel F. Noynaert, "Real-time Rate of Penetration Optimization of an Autonomous Lab-Scale Rig using a Scheduled-Gain PID Controller and Mechanical Specific Energy", IFACPapersOnLine, vol. 51, Issue 8, pp. 56-61, 2018.

[17] Dingyü Xue, Tingxue $\mathrm{Li}$, “An approach to design controllers for MIMO fractional-order plants based on parameter optimization algorithm", ISA Transactions, vol. 82, pp. 145-152, 2018.

[18] An-Ming Kang, Hong-Sen Yan, "Stability analysis and dynamic regulation of multi-dimensional Taylor network controller for SISO nonlinear systems with time-varying delay", ISA Transactions, vol. 73, pp. 31-39, 2018.

[19] Nicola Cordeschi, Danilo Amendola, Enzo Baccarelli, "Fairnessconstrained optimized time-window controllers for secondary-users with primary-user reliability guarantees, Computer Communications", vol. 116, pp. 63-76, 2018

[20] Michiel Haemers, Stijn Derammelaere, Clara-Mihaela Ionescu, Kurt Stockman, Jasper De Viaene, Florian Verbelen, "Proportional- Integral State-Feedback Controller Optimization for a Full-Car Active Suspension Setup using a Genetic Algorithm”, IFAC-PapersOnLine, vol. 51, Issue 4, pp. 1-6, 2018.

[21] Dillip Kumar Sahoo, Rabindra Kumar Sahu, G.T. Chandra Sekhar, Sidhartha Panda, "A novel modified differential evolution algorithm 
optimized fuzzy proportional integral derivative controller for load frequency control with thyristor controlled series compensator", Journal of Electrical Systems and Information Technology, vol. 5, Issue 3, pp. 944963, 2018.

[22] Yongbin Sun, Haibin Duan, Ning Xian, "Fractional-order controllers optimized via heterogeneous comprehensive learning pigeon-inspired optimization for autonomous aerial refueling hose-drogue system", Aerospace Science and Technology, vol. 81, pp. 1-13, 2018.

[23] Jiangbo Zhao, Weican Jing, Junzheng Wang, "An indirect optimization scheme for tuning a fractional order PI controller using extremum seeking”, Mechatronics, vol. 56, pp. 146-156, 2018.

[24] Sandeep Dhundhara, Yajvender Pal Verma, "Capacitive energy storage with optimized controller for frequency regulation in realistic multisource deregulated power system", Energy, vol. 147,pp. 1108- 1128, 2018.

[25] B.P. Sahoo, S. Panda, "Improved grey wolf optimization technique for fuzzy aided PID controller design for power system frequency control", Sustainable Energy, Grids and Networks, vol. 16, pp. 278- 299, 2018.

[26] Swapnil W. Khubalkar, Anjali S. Junghare, Mohan V. Aware, Amit S. Chopade, Shantanu Das, "Demonstrative fractional order - PID controller based DC motor drive on digital platform", ISA Transactions, vol. 82, pp. 79-93, 2018.

[27] Prakash Chandra Sahu, Sonalika Mishra, Ramesh Chandra Prusty, Sidhartha Panda, "Improved-salp swarm optimized type-II fuzzy controller in load frequency control of multi area islanded AC microgrid", Sustainable Energy, Grids and Networks, vol. 16, pp. 380-392, 2018.

[28] Washima Tasnin, Lalit Chandra Saikia, More Raju, "Deregulated AGC of multi-area system incorporating dish-Stirling solar thermal and geothermal power plants using fractional order cascade controller", International Journal of Electrical Power \& Energy Systems, vol. 101, pp. 60-74, 2018.

[29] B.A. Haseena, K. Srinivasan, "Development of mixed constrained RTDA controller for industrial applications", ISA Transactions, vol. 81, pp. 197209, 2018

[30] Edgar Baez, Yadira Bravo, Danilo Chavez, Oscar Camacho, "Tuning Parameters Optimization Approach for Dynamical Sliding Mode Controllers", IFAC-PapersOnLine, vol. 51, Issue 13, pp. 656-661, 2018.

[31] M.A. Ebrahim, M. Becherif, Almoataz Y. Abdelaziz, "Dynamic performance enhancement for wind energy conversion system using MothFlame Optimization based blade pitch controller", Sustainable Energy Technologies and Assessments, vol. 27, pp. 206-212, 2018.

[32] Debdoot Sain, Subrat Kumar Swain, Sudhansu Kumar Mishra, "Real Time Implementation of Optimized I-PD Controller for the Magnetic Levitation System using Jaya Algorithm", IFAC-PapersOnLine, vol. 51, Issue 1, pp. 106-111, 2018.

[33] Kangdi Lu, Wuneng Zhou, Guoqiang Zeng, Wei Du, "Design of PID controller based on a self-adaptive state-space predictive functional control using extremal optimization method", Journal of the Franklin Institute, vol. 355, Issue 5, pp. 2197-2220, 2018.

[34] Jyoti Ranjan Nayak, Binod Shaw, Binod Kumar Sahu, "Application of adaptive-SOS (ASOS) algorithm based interval type-2 fuzzy-PID controller with derivative filter for automatic generation control of an interconnected power system", Engineering Science and Technology, an International Journal, vol. 21, Issue 3, pp. 465-485, 2018.

[35] Nesrine Talbi, "Design of Fuzzy Controller rule base using Bat Algorithm", Energy Procedia, vol. 162, pp. 241-250, 2019.

[36] Shahriar Farajdadian, S.M.Hassan Hosseini, "Optimization of fuzzy- based MPPT controller via metaheuristic techniques for stand-alone PV systems", International Journal of Hydrogen Energy, vol. 44, Issue 47, pp. 2545725472, 2019.

[37] Ahmed I. Omar, Shady H.E. Abdel Aleem, Essam E.A. El-Zahab, Mostafa Algablawy, Ziad M. Ali, "An improved approach for robust control of dynamic voltage restorer and power quality enhancement using grasshopper optimization algorithm", ISA Transactions, vol. 95, pp. 110-129, 2019.

[38] Seungjae Lee, Jaewan Joe, Panagiota Karava, Ilias Bilionis, Athanasios Tzempelikos, "Implementation of a self-tuned HVAC controller to satisfy occupant thermal preferences and optimize energy use", Energy and Buildings, vol. 194, pp. 301-316, 2019.

[39] Ahmed A.S. Mohamed, Hamid Metwally, Ahmed El-Sayed, S.I. Selem, "Predictive neural network based adaptive controller for grid- connected PV systems supplying pulse-load", Solar Energy, vol. 193, pp. 139-147, 2019.

[40] M.J. Blondin, P. Sicard, P.M. Pardalos, "Controller Tuning Approach with robustness, stability and dynamic criteria for the original AVR System",
Mathematics and Computers in Simulation, vol. 163, pp. 168-182, 2019.

[41] John W. Cruz, Juan Camilo López, Daniel Dotta, Marcos J. Rider, "N-1 Multi-contingency transient stability constrained AC optimal power flow with volt/var controllers", Electric Power Systems Research, vol. 188, 2020.

[42] O. Zamzoum, A. Derouich, S. Motahhir, Y. El Mourabit, A. El Ghzizal, "Performance analysis of a robust adaptive fuzzy logic controller for wind turbine power limitation", Journal of Cleaner Production, vol. 265, 2020.

[43] Abdul Latif, S.M. Suhail Hussain, Dulal Chandra Das, Taha Selim Ustun, "State-of-the-art of controllers and soft computing techniques for regulated load frequency management of single/multi-area traditional and renewable energy based power systems", Applied Energy, vol. 266, 2020.

[44] Laxman Bhukya, Srikanth Nandiraju, "A novel photovoltaic maximum power point tracking technique based on grasshopper optimized fuzzy logic approach", International Journal of Hydrogen Energy, vol. 45, Issue 16, pp. 9416-9427, 2020.

[45] K. Jagatheesan, B. Anand, S. Samanta, N. Dey, A. S. Ashour and V. E. Balas, "Design of a proportional-integral-derivative controller for an automatic generation control of multi-area power thermal systems using firefly algorithm", IEEE/CAA Journal of Automatica Sinica, vol. 6, no. 2, pp. 503-515, 2019.

[46] M. A. Hannan, "A Quantum Lightning Search Algorithm-Based Fuzzy Speed Controller for Induction Motor Drive", IEEE Access, vol. 6, pp. 1214-1223, 2018

[47] A. Pajares, X. Blasco, J. M. Herrero and G. Reynoso-Meza, "A New Point of View in Multivariable Controller Tuning Under Multiobjective Optimization by Considering Nearly Optimal Solutions", IEEE Access, vol. 7, pp. 66435-66452, 2019.

[48] M. J. Blondin and J. P. Trovão, "Soft-computing techniques for cruise controller tuning for an off-road electric vehicle", IET Electrical Systems in Transportation, vol. 9, no. 4, pp. 196-205, 2019.

[49] A. Arefi and F. Shahnia, "Tertiary Controller-Based Optimal Voltage and Frequency Management Technique for Multi-Microgrid Systems of Large Remote Towns", IEEE Transactions on Smart Grid, vol. 9, no. 6, pp. 59625974, 2018.

[50] E. Sahin, "Design of an Optimized Fractional High Order Differential Feedback Controller for Load Frequency Control of a Multi-Area MultiSource Power System With Nonlinearity," IEEE Access, vol. 8, pp. 12327$12342,2020$.

[51] A. Ramya, M. Balaji and V. Kamaraj, "Adaptive MF tuned fuzzy logic speed controller for BLDC motor drive using ANN and PSO technique", The Journal of Engineering, vol. 2019, no. 17, pp. 3947- 3950, 2019.

[52] A. Prakash, K. Kumar and S. K. Parida, "PIDF(1+FOD) controller forload frequency control with SSSC and AC-DC tie-line in deregulated environment", IET Generation, Transmission \& Distribution, vol. 14, no. 14, pp. 2751-2762, 2020.

[53] A. K. Mishra, S. R. Das, P. K. Ray, R. K. Mallick, A. Mohanty and D. K. Mishra, "PSO-GWO Optimized Fractional Order PID Based Hybrid Shunt Active Power Filter for Power Quality Improvements", IEEE Access, vol. 8, pp. 74497-74512, 2020.

[54] G. M. Soares, P. S. Almeida, L. W. de Oliveira, J. M. Alonso and H. A. C. Braga, "Optimized Design of a Wide-Bandwidth Controller for LowFrequency Ripple Compensation in Offline LED Drivers", IEEEJournal of Emerging and Selected Topics in Power Electronics, vol. 6, no. 3, pp. 1166-1178, 2018

[55] Ruben Dario Kang, Eustaquio Alcides Martinez, Enrique Chaparro Viveros, "Coordinated tuning of power system controllers using parallel genetic algorithms", Electric Power Systems Research, vol. 190, 2021.

[56] Mara Holt, Christian Rehtanz, "Optimizing line-voltage-regulators with regard to power quality", Electric Power Systems Research, vol. 190, 2021.

[57] Wesam Taha, Abdul R. Beig, Igor Boiko, "Quasi optimum PI controller tuning rules for a grid-connected three phase AC to DC PWM rectifier", International Journal of Electrical Power \& Energy Systems, vol. 96, pp. 74-85, 2018.

[58] Amir Movahedi, Abolfazl Halvaei Niasar, G.B. Gharehpetian, "Designing SSSC, TCSC, and STATCOM controllers using AVURPSO, GSA, and GA for transient stability improvement of a multi-machine power system with PV and wind farms", International Journal of Electrical Power \& Energy Systems, vol. 106, pp. 455-466,2019. 